\title{
CONVAINCRE ET TRANSPORTER LE LECTEUR : L'HYPOTYPOSE DANS LES RÉCITS DE VOYAGES MÉDIÉVAUX CASTILLANS
}

\section{"Convincing and Captivating the Reader: Hypotyposis in Medieval Castilian Travel Narratives"}

SUMMARY - If travel narratives satisfy the reader's curiosity by providing visions of an elsewhere, offering them the illusion of a travel they cannot do, and showing them wonders, characterized by their ability to surprise, we will find those texts use hypotyposis, a "speaking painting" that develops "the art of making present the absent" and "mak[ing] the unimaginable imaginable and the improbable, probable". The development of travel narratives during the late Middle Age marked affimation of the individual and the rise of empiricism. Based on the personal experience of travellers (either real or fictional), these texts have to convince of their authenticity and transmit this direct vision (autopsiam) to make visible and palpable to the reader that unknown horizon unveiled by travelling. Hypotypose would be one of the tools erecting the travelogue as a relevant source of knowledge by sharing the traveller's sensory experience. The valuation of empirical knowledge is based on the epiphany induced by hypotyposis.

KEYWORDS - travel narratives, Castile, $15^{\text {th }}$ century, storm, Pero Tafur, Andanças, Embajada a Tamorlán

RÉSUMÉ - Si le récit de voyage donne à voir l'ailleurs pour satisfaire la curiosité des lecteurs, leur offrant l'illusion du voyage qu'ils ne peuvent faire, leur montrant les merveilles, ces objets caractérisés par leur capacité à surprendre, nous y retrouverons bien la présence de l'hypotypose, " peinture parlante » qui développe «l'art de rendre présentes les choses absentes » et de « rendre imaginable l'inimaginable et vraisemblable l'invraisemblable ». Les récits de voyages, par leur développement au cours des derniers siècles du Moyen Âge, marquèrent l'affirmation de l'individu et l'avènement de l'empirisme. Basés sur l'expérience personnelle du voyageur (qu'elle soit réelle ou fictive), il faut à ces textes transmettre et convaincre de cette vision directe (autopsiam), afin de rendre visible et palpable pour le lecteur cet horizon inconnu dévoilé par le voyage. L'hypotypose serait ainsi un des outils érigeant le récit de voyage en source pertinente de savoir par le partage de l'expérience sensible du voyageur. La valorisation du savoir empirique s'appuie sur cette caractéristique d'épiphanie de l'hypotypose.

Mots-CLÉS - récits de voyages, Castille, $\mathrm{XV}^{\mathrm{e}}$ siècle, tempête, Pero Tafur, Andanças, Embajada a Tamorlán

\section{Introduction}

Comment les récits de voyages médiévaux donnent-ils à voir l'espace parcouru ? Parviennent-ils à conférer à leurs descriptions le relief de la réalité ? Longtemps cantonnés au statut de documents historico-géographiques, ils intéressaient 
en premier lieu par leur valeur testimoniale ${ }^{1}$ et ne furent que récemment réhabilités comme objets textuels dignes d'analyse littéraire. En effet, les récits de voyages sont, en particulier, au service de la fonction mathésique qui vise à instruire le lecteur selon un modèle compilatoire et encyclopédique. Le terme désignant cette catégorie générique souligne leur dimension narrative mais celleci apparaît souvent comme un prétexte ou support permettant d'assembler, au gré de l'itinéraire, des descriptions savantes et de nombreux contenus didactiques $^{2}$ : comment ces tableaux prennent-ils place dans le rythme du texte ? Complexe agencement de narration et description, les récits de voyages ne peuvent être confondus avec de purs itinéraires ou descriptions géographiques : ces parcours sont habités, incarnés par un voyageur et leur qualité d'écriture en fait le creuset du renouveau du genre autobiographique, selon Mary B. Campbell, ou de l'éclosion du roman ${ }^{3}$. Leur capacité à convaincre de la véracité de l'expérience des voyageurs découle de la force de conviction des savoirs sur l'étranger qu'ils rapportent, mais elle permet aussi d'échapper à la liste de lieux, à l'accumulation descriptive pour gagner en tension dramatique et dynamisme. L'itinéraire et ses incidents, mais aussi les narrations interpolées provoquées par les rencontres, les anecdotes donnent aux lieux un relief absent de la simple description géographique. Mais comment ces textes concilient-ils la présence du locuteur, garant de l'authenticité du témoignage et son effacement au profit de la vivacité du récit et de l'adhésion du lecteur?

S'intéresser à la qualité d'écriture des récits de voyages relève d'un apparent paradoxe alors qu'on leur reproche fréquemment de n'être que de simples carnets de route, terne accumulation de descriptions. Les auteurs eux-mêmes n'en viendraient à prendre la plume qu'à la faveur d'un concours de circonstances auquel leur formation ou plutôt leur absence de formation ne les aurait pas préparés : des personnages «illettrés », davantage tournés vers les intérêts pragmatiques du commerce et ne se distinguant justement que par leur expérience de voyageurs. Dépourvus de l'érudition nécessaire à la rédaction d'un texte de qualité, pour atteindre un degré de rédaction acceptable, les voyageurs devraient

\footnotetext{
${ }^{1}$ J. Richard, « Voyages réels et voyages imaginaires, instruments de la connaissance géographique au Moyen Âge », in : idem, Croisés, missionnaires et voyageurs. Les perspectives orientales du monde latin médiéval, London, Variorum, 1983, p. XX ; J. Ochoa Anadón, "El valor de los viajeros medievales como fuente histórica", Revista de Literatura Medieval 2 (1990), p. 85-102 ; J. Richard, "La relation de voyage, instrument de la transmission du savoir géographique », in: La Transmission des savoirs au Moyen Âge et à la Renaissance, sous la dir. de Pierre Nobel, Presses Universitaires de Franche-Comté, 2005, p. 303-318.

${ }^{2}$ Nous distinguons les descriptions ornementale, expressive et représentative, et, au sein de cette dernière, les fonctions mathésique, mimésique et sémiosique. La fonction mathésique fournit des savoirs et sert l'entreprise didactique. Elle est généralement liée à l'emploi d'un vocabulaire spécialisé ; J.-M. Adam et A. Petitjean, Le Texte descriptif : poétique historique et linguistique textuelle, Paris, Nathan-Université, 1989, p. 122.

${ }^{3}$ M.B. Campbell, The Witness and the Other World : Exotic European Travel Writing, 400-1600, Ithaca Cornell University Press, 1988.
} 
avoir recours à une plume experte comme dans le cas de Marco Polo et du rédacteur lettré à qui il devrait son succès ${ }^{4}$.

En opposition avec de tels préjugés, nous analyserons ici les seuls deux réels récits de voyages castillans que le Moyen Âge a conservés. Andanças e viajes de Pero Tafur fut rédigé après 1455, d'après un voyage réalisé de 1436 à 1439, à titre strictement personnel, par un noble sévillan ${ }^{5}$. Ce récit semble particulièrement propice à l'étude de cette volonté de mise en scène car il a pour but de valoriser l'auteur, en narrant ses prouesses et les dangers affrontés, d'affirmer sa haute noblesse, son courage et l'affection que lui porteraient les plus grands personnages de son temps. D'autre part, Embajada a Tamorlán ${ }^{6}$ est le compte rendu de la mission diplomatique réalisée entre 1403 et 1406 et envoyée par Henri III (1379-1406) à Tamerlan, souverain d'origine mongole et descendant de Gengis Khan. Les ambassadeurs, après avoir longtemps attendu pour rencontrer Tamerlan, n'en obtinrent pas la réponse espérée et sans doute le récit avait-il aussi pour but de pallier l'insuccès de leur mission.

Si le récit de voyage donne à voir l'ailleurs pour satisfaire la curiosité des lecteurs, leur offrant l'illusion du voyage qu'ils ne peuvent faire, nous y retrouverons bien la présence de l'hypotypose, « peinture parlante » qui développe "l'art de rendre présentes les choses absentes » et de « rendre imaginable l'inimaginable et vraisemblable l'invraisemblable $»^{7}$. Quels moyens sont mis en œuvre par les auteurs pour déployer l'enargeia, la vivacité, la force de conviction nécessaire pour emporter l'adhésion du lecteur et lui donner à voir ce monde étrange ? Cette écriture mêlant description des territoires étrangers et narration de l'expérience du voyageur devrait donc nous donner à lire de multiples variations qu'il nous faudra comparer entre hypotypose, ekphrasis, récit dramatique... Les récits de voyages, par leur développement en Occident à partir du $\mathrm{XV}^{\mathrm{e}}$ siècle, marquèrent l'affirmation de l'individu et l'avènement de l'empirisme. Basés sur l'expérience

\footnotetext{
${ }^{4}$ La réception faussée de Marco Polo tient sans doute en partie à son processus de rédaction. Marco Polo, emprisonné à Gênes, sollicite l'aide de Rustichello de Pise. De ce dernier on connaît une compilation en prose d'aventures arthuriennes, Meliadus. Les contenus transmis par Marco Polo nécessitaient une mise en forme littéraire. Les versions les plus anciennes présentent des traits de rhétorique chevaleresque. La comparaison du Meliadus avec le chapitre XV de Marco Polo en souligne les similitudes. J. Larner, Marco Polo y el descubrimiento del mundo, Barcelona, Paidós, 2001.

${ }^{5}$ P. Tafur, Andanças e viajes, ed. M.Á. Pérez Priego, Fundación José Manuel Lara, clásicos andaluces, Sevilla, 2009.

${ }^{6}$ Embajada a Tamorlán, in : Viajes medievales, II, ed. M.Á. Pérez Priego, Fundación José Antonio de Castro, Madrid, 2006. Cf. F. López Estrada, "Fama literaria de Tamorlán en España durante el siglo XV", in : Studia in Honorem Germán Orduña, Alcalá de Henares, Universidad de Alcalá de Henares, Servicio de Publicaciones, 2001, p. 369-374.

${ }^{7}$ M. Fumaroli, L'Âge de l'éloquence [Droz, 1980], Paris, Albin Michel, Bibliothèque de l'Évolution de l'Humanité, 1994, p. 678-679. Voir aussi : C. Ginzburg, Le Fil et les traces. Vrai faux fictif, trad. par M. Rueff, Lagrasse, Verdier Histoire, 2006. B. Boulay, « Effets de présence et effets de vérité dans l'historiographie ", Écrire l'histoire, Littérature, $\mathrm{n}^{\circ}$ 159, 2010, p. 26-38; URL: http://www.jstor.org/stable/41705312; consulté le 26 juillet 2016.
} 
personnelle du voyageur (qu'elle soit réelle ou fictive), il faut que ces textes transmettent et convainquent de cette vision directe (autopsiam), afin de rendre visible et palpable pour le lecteur (parousian) cet horizon inconnu dévoilé par le voyage. L'hypotypose serait ainsi un des outils érigeant le récit de voyage en source pertinente de savoir par le partage de l'expérience sensible du voyageur. La valorisation du savoir empirique s'appuie sur cette caractéristique d'épiphanie de l'hypotypose ${ }^{8}$.

\section{Le vraisemblable, le tableau et les détails}

Le tableau descriptif s'abstrait du fil narratif pour imposer un autre rythme à la lecture mais il n'est pas immobile : l'hypotypose parvient justement à insuffler de la vivacité à la description, à emporter le lecteur dans un mouvement soutenu. Ce rythme spécifique trouverait sa clef dans l'abondance de détails, l'exhaustivité, l'énumération... Selon Yves le Bozec, la «principale qualité rhétorique de l'ekphrasis est sa capacité d'exhaustion ${ }^{9}$; Barbara Cassin évoque quant à elle «une mise en phrase qui épuise son objet» et explique le terme comme $e k$-, (jusqu'au bout), et phrazo, (faire comprendre, montrer, expliquer) ${ }^{10}$. Qu'en est-il de l'hypotypose ? S'il lui faut donner à voir, elle requiert également deux qualités rhétoriques essentielles : l'économie (" éviter une totale prolixité »), et le vraisemblable. La force de conviction de l'hypotypose suppose le vraisemblable qu'un excès de détails pourrait détruire. Ainsi le narrateur doit sélectionner les éléments pertinents, propres à marquer l'imagination ou essentiels à l'objet décrit. Pero Tafur insiste de la sorte fréquemment sur la nécessité de sacrifier certaines informations pour ne pas perdre la confiance de son lecteur ${ }^{11}$. Le narrateur reste présent dans les descriptions dont il affirme de diverses manières la véracité, ne serait-ce que par les prétéritions en référence aux doutes que pourraient émettre les futurs lecteurs.

Les détails descriptifs servent à convaincre de la véracité du récit mais aussi à donner vie à la scène, à y immerger le lecteur. L'indication de distance permet à Tafur de souligner l'immédiateté de son agression sur les routes près de

\footnotetext{
${ }^{8}$ « Enargeia est donc bien le mode privilégié de la manifestation sensible, l'effet de la connaissance empirique immédiate, le critère de la vérité objective, essentiellement par le moyen de la vue. Inséré dans une théorie de la connaissance, le concept est par excellence le moyen qui permet de faire l'économie du recours au langage. Concept anti-rhétorique s'il en est !». C. Calame, «Quand dire, c'est faire voir, l'évidence dans la rhétorique antique », Études de lettres, Faculté des Lettres, Université de Lausanne, ${ }^{\circ}$ 4, 1991, p. 3-22.

${ }^{9}$ Y. Le Bozec, «L'hypotypose : un essai de définition formelle », L'Information Grammaticale, $\mathrm{n}^{\mathrm{o}} 92,2002$, p. 3.

${ }^{10}$ B. Cassin, L'Effet sophistique, Paris, Nrf, Essais, Gallimard, 1995, p. 501 et 680.

${ }^{11}$ P. Tafur, Andanças e viajes, op. cit., p. 94 : « la caravana llegó la cual traía muy muchos camellos tantos que yo no lo escrivó porque no paresca fablar demasiado ».
} 
Vienne, sa surprise d'être attaqué immédiatement après avoir quitté ses compagnons : «E no fui apartado de ellos cuanto media legua, cuando me saltearon en el camino a pie ciertos fidalgos pobres de la tierra por me robar $[\ldots] »^{12}$. On constate, par cet exemple, que la mesure de la distance permet l'expression indirecte du temps et devient un des éléments au service de l'expressivité, donnant à ressentir la surprise et la détresse du voyageur, une détresse de courte durée : les actions s'enchaînent sans même que la phrase ne s'interrompe, donnant le rythme de la fuite rapide et efficace de Pero Tafur et ses compagnons, fuite permise par la qualité des montures : «[...] pero no podieron fazer nada, que yo e mis hombres traiemos Buenos cavallos e asíme fui a la cibdad a posar do me avían señalado $»^{13}$. Suite à cette agression, l'épisode se clôt par un retournement improbable : alors qu'il s'est installé pour déjeuner à l'auberge, ses agresseurs $\mathrm{y}$ arrivent et s'engage alors une conversation au style indirect qui instaure une complicité inattendue entre nobles peu fortunés et en situation de fragilité. La polysyndète énumère les actions suivantes en un flux rapide et expéditif, transformant les agresseurs en amis qui lui offrent leurs services (contre rétribution) : " ellos me respondieron que, pues que asi era, que les perdonase e que ellos querían ir a buscar para ellos e para mí. E yo agradezigelo mucho e fizeles comer conmigo, e diles sendos florines e fueron mucho contentos e, tanto que en la cibdad estuve, los más días me acompañavan $»^{14}$. Si le retournement et la « transmutation » de ces gentilshommes offrent une péripétie divertissante, cela met surtout en exergue le talent de Pero Tafur pour tirer parti des situations avec une diplomatie pragmatique. L'épisode qui vante de façon allusive la présence d'esprit et la capacité à convaincre du héros, ouvre le chapitre sur Vienne qui se prolonge par la description détaillée du paysage et de l'architecture qu'il découvre ainsi escorté.

Pero Tafur sait également avoir recours à la mise en scène et aux métaphores expressives pour communiquer avec vivacité sa vision d'un événement surgissant comme un tableau dynamique propre à emporter l'adhésion du lecteur. Cette narration théâtralisée est particulièrement nette pour les fêtes nautiques de Venise, ce théâtre sur l'eau qui déploie en grande pompe les noces de la mer avec les Vénitiens : «dizen ellos que ésta es una cirimonia antigua que desposan a la mar con la tierra esto por aplacar su furia». Durant cette fête décrite avec une emphase admirative, les embarcations sont si nombreuses que,

\footnotetext{
${ }^{12}$ Ibid., p. 233 ; « et je ne m'étais pas éloigné d'eux d'une demi-lieue, lorsque je fus attaqué sur le chemin à pieds par certains gentilshommes pauvres du pays qui voulaient me voler ». Je proposerai mes propres traductions en notes infrapaginales pour l'ensemble de ce travail.

${ }^{13} \mathrm{Ibid} . ;$; [...] mais ils ne purent rien faire car moi et mes hommes avions de bons chevaux et c'est ainsi que je parvins à la ville pour loger où on m'avait conseillé de le faire ».

${ }^{14} \mathrm{Ibid}$; ; «[...] ceux-ci me répondirent que, puisqu'il en était ainsi, ils demandaient mon pardon et qu'ils voulaient s'efforcer et pour eux et pour moi-même. Et moi je les remerciai fort et je les fis manger en ma compagnie et je leur donnai à chacun quelques florins et ils en furent très contents et, tant que je fus dans cette ville, ils m'accompagnaient la plupart du temps ».
} 
par hyperbole, « la mer paraît avoir disparu $»^{15}$. Ici la formulation généralise un point de vue particulier au présent de l'indicatif propre à actualiser la scène, à immerger le lecteur / spectateur dans cette foule et ce décor. La description de la procession maritime en retranscrit toutes les différentes étapes et prétend à l'exhaustivité à travers de nombreuses incises: "Este día sale el duce en toda su magnificencia e cirimonias papales e impériales, que dizen que en tal día las ganó, e fazen grandes procesiones e, oída la misa, sale con toda la clerecía e va a la mar ». La succession d'actions est retranscrite en longues énumérations grâce à la polysyndète, accélérant le rythme comme si le regard peinait à suivre les mouvements des nombreux navires qui convergent. Les détails rapportés couvrent des éléments variés comme la nature des embarcations, les décors, les mesures précises, les acteurs... ou les termes italiens comme ici «Vicentoro", en réalité bucintoro qui désigne une embarcation (buzo : ancien navire vénitien de guerre et de commerce) recouverte de décorations en or : "van en una fusta que se llama Vicentoro e es un tercio más larga que una galea e dos tantos más ancha e los que vogan van so sota, que no parecen, e va toda toldada de muy ricos panos de oro e el suelo de buena tapetería ». Les comparaisons de longueur et largeur avec une galère, celles désignant les tapis et voilages, permettent au lecteur de mieux se figurer les éléments originaux ou exotiques ${ }^{16}$. Au final, dans ce tableau la médiation du locuteur s'efface et l'écriture fait spectacle pour sidérer le lecteur en lui transmettant l'essentiel qui est bien ici la vision : «es una fermosa cosa de ver ».

Après ce tableau de Venise, regardons son pendant plus loin dans le même récit, la Venise du Nord. La peinture laudative de Bruges par ce noble qui n'a pas honte de manier l'argent ni d'être proche des milieux commerçants offre un morceau de bravoure plein d'emphase exclamative, d'hyperbole, bref, d'un enthousiasme contagieux. Cette ville, principal centre commercial de l'Europe occidentale, apparaît comme un concentré de ce que le monde peut offrir de mieux à condition d'avoir l'argent pour se l'offrir à travers une anaphore répétant l'adverbe de lieu, le verbe voir et les objets de désirs que procurent les réseaux commerciaux :

\footnotetext{
${ }^{15}$ Ibid., p. 173 ; « quant à eux, ils disent que cette cérémonie antique opère la mariage de la terre avec la mer afin d'apaiser la furie de cette dernière ». «E parece que la mar ne se dexa ver, tan llena va de fustas » (« il semble que la mer ne se laisse plus voir tant elle est pleine d'embarcations »).

${ }^{16}$ C'est ce que dénote le double mouvement contradictoire qui préside à l'écriture du voyage selon R. Le Huenen : « une ouverture vers le réel, vers la nouveauté qui constitue le principe moteur du voyage, et une appropriation du réel par voie d'analogie qui en est le principe régulateur. S'il affecte de s'ouvrir à l'inouï, à l'étrangeté du référent, le discours du voyageur s'empresse de reconstruire le monde selon un modèle connu, de réduire les écarts et les différences et de projeter sur la réalité nouvelle le moule d'un sens déjà connu ». R. Le Huenen, "Qu'est-ce qu'un récit de voyages? », in: Les Modèles du récit de voyages, Litterales $\mathrm{n}^{\circ}$ 7, 1990, p. 18.
} 
e ciertamente quien gran dinero toviese e voluntad de lo despender, bien fallaríe allí sola en aquella cibdad lo que por todo el mundo nace. Allí vi las naranjas e las limas de Castilla que parecen que entonces las cogen del árbol. Allí las frutas e vinos de la Grecia, tan abondosamente como alla. Allí vi las confaciones e especerias de Alexandría e de todo el levante como si alla estoviera. Allí vi las pelleterias del Mar mayor como si allí nacieran. Allí estava toda Italia con sus brocados e sedas e arneses e todas las otras cosas que en ella se fazen. Así que no ay de parte del mundo cosa donde allí no se fallase lo mejor que en ella ay $[\ldots]^{17}$.

Ce qu'offre à voir cette énumération fascinante, ce lyrisme matérialiste, c'est l'illusion créée par l'action acharnée des commerçants (avec les verbes au subjonctif imparfait exprimant l'irréel passé : «estoviera », «nacieran »), l'abondance de ces produits réunis niant l'éloignement des territoires et leurs spécificités géographiques. Le locuteur emmène le lecteur au contact de cet éden, de ce pays de Cocagne qui à lui seul résume tous les voyages qui pourraient être réalisés... mais à quoi bon voyager quand le meilleur des produits vous est apporté par les commerçants et que la description des espaces vous est transmise par les auteurs?

\section{Persuader et séduire : la fonction émotive}

En créant l'émotion de l'allocuteur, l'hypotypose a toujours une valeur de persuasion et de séduction. Elle l'amène à suspendre son jugement ${ }^{18}$. Cette fonction émotive apparaît avec force dans les épisodes où le narrateur-voyageur est en danger et cherche à partager ces sentiments violents, cette sidération de l'instant avec le lecteur. L'espace de la mer, caractérisé par le sublime et le danger, est particulièrement propice à ces tableaux violents et superbes ${ }^{19}$. Dans

\footnotetext{
${ }^{17}$ P. Tafur, Andanças e viajes, op. cit., p. 212 ; « et certainement celui qui aurait beaucoup d'argent et le désir de le dépenser, trouverait bien là, dans cette seule ville, tout ce qui nait à travers le monde. Là, j'ai vu les oranges et les citrons de Castille qui paraissent à peine cueillis de l'arbre. Là, les fruits et les vins de Grèce, tout aussi abondant que là-bas. Là, j'ai vu les confiseries et les épices d'Alexandrie et de tout le Levant comme si j'y avais été. Là, j'ai vu les peausseries de la mer Noire comme si c'était là qu'elles étaient nées. Là, était toute l'Italie avec ses brocards, ses soieries, ses harnais et toutes les choses qui s'y fabriquent. Ainsi il n'y a pas de chose d'où que ce soit au monde qui ne se trouve là, et de la meilleure qualité ».

${ }^{18}$ L'hypotypose fait jaillir « un enthousiasme et un mouvement extraordinaire de l'âme »; Traité du sublime, trad. de N. Boileau, éd. de F. Goyet, Paris, Le Livre de Poche, 1995, p. 97. «L'esprit de l'auditeur est aisément entraîné par cette Image qu'on lui présente au milieu d'un raisonnement ; et qui lui frappant l'imagination, l'empêche d'examiner de si près la force des preuves, à cause de ce grand éclat dont elle couvre et environne le discours »; Y. Le Bozec, op. cit., p. 5.

${ }^{19}$ La tempête, le naufrage lui-même étaient des éléments topiques des récits de pèlerinages, encore plus développés dans les récits de voyages. C. Buchet et C. Thomasset (éd.), Le Naufrage. Actes $d u$ colloque tenu à l'Institut Catholique de Paris (28-30 janvier 1998), Paris, Honoré Champion, 1999. J.M. Herrero Massari, "El naufragio en la literatura de viajes peninsular de los siglos XVI y XVII", Revista de Filología Románica 2.14 (1997), p. 205-213. C. Deluz, « Pèlerins et voyageurs face à la
} 
la Embajada a Tamorlán, les différents obstacles rencontrés sont développés et dramatisés pour mettre en valeur le courage et l'obstination des ambassadeurs dans l'accomplissement de leur mission qui aurait pu plus d'une fois leur coûter la vie. La première partie du récit est donc une véritable odyssée où se succèdent les tempêtes, un naufrage et les menaces induites par les conflits opposant les Génois, les Turcs et les Catalans. Face à cela, l'auteur dépeint le héros collectif des ambassadeurs en un récit originalement réalisé à la troisième personne du pluriel. La distanciation induite renforce l'impression d'objectivité du récit diplomatique.

Le narrateur de la Embajada aurait pu rapidement évacuer le récit de la navigation au travers de la Méditerranée, un espace bien connu et dépourvu d'informations relatives à leur mission auprès de Tamerlan. Cependant, cette narration d'une traversée agitée est pleinement développée, alternant moments de dangers et d'angoisses et épisodes heureux sur les îles florissantes de la Méditerranée. La tension dramatique culmine dans les tempêtes au cours desquelles le narrateur prête une attention fine aux phénomènes naturels, aux mutations de la mer et du ciel, aux bruits et lumières. La vision d'une tornade s'incarne dans un passage où se multiplient les verbes d'actions pour détailler le mouvement ascendant de l'eau vers un ciel obscurci et les réactions affolées des marins et passagers. Les comparaisons à des branches et de la fumée, les superlatifs, la polysyndète confèrent leur force à la narration :

A ora de vísperas vieron descaer del cielo dos ramos como de fumo que llegaron hasta el mar y el agua subió por ellos tan aína e tan recio con gran ruido que las núes finchó de agua, y escureció y anubló el cielo y arredráronse con la carraca cuanto podieron, ca dezían que si aquellos ramos acertaran a tomar la carraca que la podrían anegar ${ }^{20}$.

La mention d'une bonace inquiétante («andovieron entre dichas dos islas que no podían salir de ellas por calma que fazía ») prépare le lecteur à l'irruption d'un nouveau tableau de tempête. L'impact dramatique de la tempête est encore augmenté par le spectacle offert par les volcans en activité dans les îles Éoliennes $^{21}$. Face à ces deux jours de tempête ininterrompue, le capitaine du navire,

mer, $\mathrm{XII}^{\mathrm{e}}-\mathrm{XVI}^{\mathrm{e}}$ siècles ", in : Horizons marins, itinéraires spirituels, H. Dubois, J.-C. Hocquet et A. Vauchez (dir.), t. 2, Marins, navires et affaires, Paris, Sorbonne, 1987, p. 277-288.

${ }^{20}$ Embajada a Tamorlán, op. cit., p. 15 ; «à l'heure de vêpres, ils virent descendre du ciel deux branches comme de fumée qui arrivèrent jusqu'à la mer et l'eau monta par ceux-ci si vite, si fort et avec un grand bruit que la nuée se gonfla d'eau, et le ciel s'assombrit et ils se pressèrent avec leur caraque autant qu'ils le purent car ils disaient que si ces branches réussissaient à prendre la caraque, ils pourraient la noyer $»$.

${ }^{21}$ «[...] rompió las velas de la carraca y anduvieron a árbol seco de una parte a otra de manera que se vieron en gran peligro. E duró la dicha tormenta martes y miércoles fasta dos horas de la noche e las dichas bocas, señaladamente la de Strangol y Bolcante, con el gran viento lançava grandes llamas de fuego y fumo con gran ruido, y durante la tormenta fizo el patrón cantar las ledanías e que todos pidiessen misericordia a Dios ». Ibid., p. 16; « les voiles de la caraque se 
tout aussi inquiet que le reste de l'équipage, ordonne une prière collective ( «Fizo el patrón cantar las ledanías e que todos pidiessen misericordia a Dios ${ }^{22}$ ) à laquelle se joignent les ambassadeurs. Mais, à peine est-elle achevée que des lumières étranges et inexpliquées apparaissent. Le narrateur souligne la valeur du témoignage direct collectif par l'utilisation du verbe « ver » répété avec insistance tout au long de ce paragraphe, comme pour imprimer en retour cette vision à la rétine du lecteur. La mention de la durée est également répétée, avec en outre la comparaison avec la messe comme référent («Estas lumbres vieron cuantos estavan en la carraca, que fueron llamados que las viniessen ver y duraron una pieça» : «tout ceux qui étaient sur la caraque virent ces lumières et elles durèrent un long moment »; "y duraron estas lumbres cuanto dura una missa » : « et ces lumières durèrent le temps d'une messe). Le narrateur insiste sur la simultanéité de ces phénomènes (avec en particulier une expression redoublée en chiasme : «E no cessava en todo esto la tormenta » et «y en todo esto la tormenta no cessava »). En plus des lumières des voix mystérieuses résonnent («voces como de omes $»$ : des voix comme d'hommes). Rien ne manque à ce tableau pittoresque des dangers de la mer face auxquels les marins et leurs passagers ne peuvent s'en remettre qu' au pouvoir de la prière, dernier recours qui souligne la peur ressentie par les ambassadeurs face à cette nature déchaînée.

Les péripéties s'enchaînent à un rythme soutenu. Résumant les journées de navigation sereine, la narration s'étend bien davantage sur les tableaux consacrés aux périls, construits selon une trépidante accumulation d'incidents. Le navire, après avoir touché un haut fond, perd l'usage de son gouvernail. La scène est décrite dans une phrase longue à en perdre le souffle (quinze lignes !), sans laisser de répit au lecteur, mais le navire s'en sort ${ }^{23}$. Le narrateur collectif de l'ambassade (troisième personne du pluriel sur l'ensemble de l'œuvre) est particulièrement peu présent dans ces passages maritimes et les rares mentions personnelles paraissent recouvrir un autre personnage collectif, celui de l'ensemble des hommes embarqués sur le même navire et soumis à la même inquiétante menace des éléments. Cet effacement du locuteur permet une plus grande objectivation des scènes, décrites avec une efficacité de moyens où seul l'élément le plus frap-

déchirèrent et ils durent fuir à sec de toiles d'un bout à l'autre de sorte qu'ils se virent en grand danger. Et la tempête dura mardi et mercredi jusqu'à deux heures du matin, et lesdites bouches, précisément Stromboli et Vulcano, avec le grand vent lançaient de grandes flammes de feu et de la fumée avec grand bruit, et pendant la tempête, le capitaine leur fit prier à tous les litanies et implorer la miséricorde de Dieu ». Les éruptions régulières des îles volcaniques Vulcano et Stromboli, jusqu'à plusieurs par heure, visibles de très loin la nuit, expliquent leur surnom de « phare de la Méditerranée ».

${ }^{22}$ Ibid., p. 16-17.

${ }^{23}$ «En la noche creció el viento [...] tocó la carraca en tierra y saltó el timón de caxa e ovieron de ser perdidos salvo el viento que era poco ». Ibid., p. 17 ; « durant la nuit le vent vint à forcir [...] la caraque toucha terre et cela fit sauter le timon de sa caisse et ils auraient été perdus si le vent avait été plus fort ». 
pant suffit pour esquisser la péripétie ou plutôt la détresse des voyageurs face à la succession des péripéties. À nouveau mis en danger par un courant violent, leur navire passe si près d'une côte rocheuse que les passagers peuvent entendre piailler des faucons dans leur nid et que certains marins et le pilote lui-même se préparent à nager ${ }^{24}$. On note à la fois comment le détail concret, prosaïque mais extraordinaire donne à sentir la proximité du danger et la prédilection du narrateur pour l'élément auditif dans ces descriptions totales de périls maritimes. C'est la grâce de Dieu que le narrateur remercie encore une fois en dénouement et le même appel à la grâce divine se retrouve lors du voyage du retour des ambassadeurs, leur ancre ne parvenant pas à les fixer. Ce tableau de tempête nous donne à voir une caraque ballotée et qui finalement fait naufrage, sa mâture risquant de briser leur galiote, un grand péril évité in extremis, dénouement heureux vers lequel le lecteur est conduit par une longue phrase fragmentée accumulant les détails et les menaces :

El mar creció mucho y el viento era recio e la tormenta alta e, cuando pensaron tornar al puerto do avían partido, no podieron e,desque vieron que no podieron llegar a la carraca ni tornar al puerto, echaron dos anclas. E la tormenta creció todavía e traía las anclas atanto que echó la galeota entre unas rocas e quiso Nuestro Señor Dios que las anclas araron tanto a que la galeota salvó las rocas sin tocar en ellas, ca si tocara luego fuera deshecha e entonces tovieron las anclas que no araron e la tormenta creció en tanta manera que era espanto e todos se encomendavan a Nuestro Señor Dios que pensavan nunca escapar ${ }^{25}$.

Le narrateur emploie de nombreux termes spécifiques de marine tant pour décrire les manœuvres de marins que les avaries subies ou les mouvements désordonnés du navire qui fait eau, traversé par les vagues ${ }^{26}$.

\footnotetext{
${ }^{24}$ Les pronoms comparatifs soulignent l'intensité du danger : «No podieron tomar la vuelta tan aína que la dicha carraca no passó tan cerca de tierra que unos falcones pequeños que criavan en una peña sonavan e viéronse en peligro, de manera que el nauchel e algunos mercaderes y marineros se desnudaron en jubones y cuando fueron longados entendieron que Dios les avía hecho mucha merced ». Ibid., p. 18 ; « ils ne purent prendre le virage avec une vivacité suffisante pour éviter que ladite caraque ne passe si près de la terre qu'on entendit à bord les cris de certains faucons juvéniles qui nichaient sur un rocher et le navire se vit en grand danger, de sorte que le pilote et quelques marchands et marins se déshabillèrent et lorsqu'ils se furent éloignés ils comprirent que Dieu leur avait fait une grande grâce ».

${ }^{25} \mathrm{Ibid}$., p. 60 ; «la mer forcit beaucoup et le vent devint plus vif et la tempête était à son maximum et, alors qu'ils projetaient de revenir au port dont ils étaient partis, ils ne le purent point, et, constatant qu'ils ne pouvaient arriver à la caraque ni rentrer au port, ils jetèrent deux ancres. Et la tempête devint plus forte encore, et elle traînait les ancres de telle manière qu'elle jeta la galiote entre des rochers, et Notre Seigneur voulut bien que les ancres raclèrent tant le sol que la galiote fut sauvée sans toucher les rochers, car si elle les avait touchés, en un instant elle aurait été brisée, et alors les ancres ne raclèrent plus et la tempête devint si forte que cela était effroyable et tous se recommandaient à Notre Seigneur Dieu car ils pensaient ne pas s'en sortir ».

${ }^{26}$ «E las vagas fazían tan altas que quebravan e entravan por el un borde e salian por el otro $e$ la galeota trabajava mucho e fazía mucha agua », ibid.; " et les vagues étaient si hautes qu'elles se
} 
Ces tempêtes sont des moments clefs de la narration où les voyageurs, aux prises avec une nature grandiose, perdent le contrôle de leurs mouvements et sont submergés d'émotions que peuvent partager les lecteurs entraînés à leur tour dans le flux du texte qui accumule les verbes d'actions. Un net changement de rythme fait que ces épisodes se singularisent. Avec l'effet de surprise, les ruptures propres à émouvoir, on sort de la description panoramique. Surgissent les verbes d'action, les polysyndètes, les superlatifs, l'intensité du récit avec l'allongement des phrases composées de nombreux segments courts, pressés, uniquement reliés par la répétition hypnotique de la conjonction « $e$ ».

Plus encore peut-être que la Embajada, le récit de Pero Tafur révèle le soin apporté à la mise en scène de la narration, les multiples détails et personnages étant utilisés pour donner le plus de relief possible à l'anecdote de cette course poursuite maritime qui enchaîne les péripéties et happe le lecteur. À son retour à Rhodes, le navire sur lequel est embarqué Pero Tafur est poursuivi par une galère turque dont les intentions hostiles sont justifiées par un désir de vengeance suite à une agression catalane ${ }^{27}$. Dans cet épisode, le lecteur est plongé dans la réalité des manœuvres et des dangers par la précision du vocabulaire nautique. De nombreux procédés dynamisent le tableau de cette course poursuite, accélèrent le rythme, la scansion se calquant sur la marche forcée du navire, la difficulté à distancer le poursuivant. Il leur faut en effet avancer à la rame pour gagner de vitesse la galère turque et l'équipage doit fournir pour cela un effort exceptionnel exprimé par la mention de leur chant et la synecdoque de leurs mains fatiguées $^{28}$. Ici, comme dans les scènes de tempête dans la Embajada, le personnage collectif désigne bien plus l'ensemble des personnes embarquées et le narrateur paraît s'effacer derrière la prégnance du jeu d'opposition des pronoms «nous » contre « eux ». S'ils parviennent à prendre une vitesse considérable, c'est cependant indirectement grâce à Tafur puisque le maître d'équipage catalan à l'origine de cette prouesse lui doit la vie. Il l'a en effet sauvé de la pendaison, après que la corde a rompu, ce que Tafur interprète comme un signe divin qu'il s'agit de ne pas ignorer. Dieu remercie donc Pero Tafur d'avoir pris en compte ce signe et sauvé la vie de ce maître d'équipage qui possède l'art de la navigation et allège le navire juste à temps pour distancer les Turcs ${ }^{29}$. Le navire ami qui les accompagne

\footnotetext{
brisaient et entraient par un bord et sortaient par l'autre et la galiote travaillait beaucoup et prenait l'eau en quantité ».

${ }^{27}$ P. Tafur, Andanças e viajes, op. cit., p. 115 : «e antes de dos oras vimos venir una galea de turcos que venía contra nosotros para nos tomar e despedaçar por una fusta que los catalanes avían tomado de turcos sobre el puerto de Chipre ».

${ }^{28}$ «E nosotros a vela e a remos e ellos asimismo, tanto que allí no fallecía la letania, las manos bien trabajadas de remar ». Ibid., p. 115.

${ }^{29}$ "Iva comigo un cómitre de una galea de catalanes [...] e quebrose la soga con é. E yo roguele tanto que me lo dies, pues Dios avía fecho tanto por él, e plúgole e por esto escapamos, que sabía mucho de navegar. Fizo aliviar el peso que levava nuestro gripo para que mejor caminase e el otro de mercadurías no quiso echarlo a la mar ».
} 
refuse de perdre ses marchandises pour prendre de la vitesse et est donc rattrapé, cet échec permettant d'exprimer plus lourdement la menace qui pesait sur leur propre navire. Le temps que perdent les Turcs à attaquer ce navire et à massacrer son équipage aurait permis au navire de Pero Tafur de prendre de l'avance. Ici le texte est rythmé par les allusions au soleil comme repère temporel, mais sa course dans le ciel semble suspendue, comme gelée dans la précipitation des événements : il « veut» puis «a voulu » se coucher; une suspension qui transcrit un état de sidération, le ralentissement paradoxal d'une action trop intense («e cuando ya era tarde, que el sol se querie poner [...] ovimos tiempo de alargarnos un poco e, Cuando quiso anochecer, guindamos la vela cuanto pudimos e, cuando fue noche oscuro »). Ayant hissé les voiles « autant qu'ils le pouvaient », ils se retrouvent à croiser sans un bruit le navire ennemi, une scène dont la description cherche à transcrire la tension alors que tous les passagers doivent prendre les rames, Tafur soulignant ainsi sa participation active à cette fuite aussi silencieuse que possible ${ }^{30}$.

Mais la tension ne retombe par pour autant, ou du moins l'auteur ne le permet pas et enchaîne à nouveau les péripéties. C'est alors une tempête qui les assaille alors que la menace de la galère turque n'est toujours pas écartée, car leur navire plus lent risque à tout moment d'être rattrapé. C'est par une exclamation héroïque que le narrateur annonce qu'ill préfère mourir de la main des Turcs plutôt que noyé. Une nouvelle péripétie surgit alors puisque Tafur doit intervenir pour sauver la vie d'un de ses hommes ${ }^{31}$. La conclusion donnée à l'épisode exprime le repos enfin atteint par le narrateur et compare l'aventure vécue à une maladie tant il en sort brisé, ce qu'il souligne encore à plusieurs reprises dans les pages suivantes ${ }^{32}$, nous rappelant que les péripéties sont avant tout les épreuves valorisant le héros chevaleresque. La lecture détaillée de ce pittoresque épisode maritime démontre la volonté de transporter le lecteur par le rythme enlevé, la succession des péripéties, les détails les plus dramatiques comme l'expression du danger et la mention des multiples personnages impliqués. Toutefois on peut s'interroger : s'agit-il d'une hypotypose au vu de la présence du héros narrateur? Ici il est moins héros que spectateur impuissant, fenêtre ouverte pour le lecteur vers une réalité dont il transmet l'expérience. En effet le narrateur balloté par les flots n'a pas prise sur l'action, n'agit pas face aux évé-

\footnotetext{
${ }^{30}$ «Todos tomamos mano a los remos e trabajamos media ora cuanto en el mundo podimos e, cuanto fue noche oscuro, calamos la vela [...] bogando muy quedo que no sonasen los remos e la galea pasó bien cerca de nosotros que no nos vido ».

31 «E a medianoche saltó un viento a la mar de mediodía, que cada onda nos envistie de banda a banda ¡Cuánto yo más quisiera ayer caído en poder de los turcos que no ser anegado en tal lugar! Alli me querian echar un ombre mío a la mar salvo que lo defendimos muy bien. [...] E allí reposamos como quien escapa de una gran dolencia », ibid., p. 116.

32 «Con gran miedo [...] yo me pensava moror según el trabajo que avía pasado », ibid.
} 
nements et décrit seulement les obstacles comme les solutions que tente de proposer l'équipage. Cette position de spectateur fait tableau, maintient le texte dans la description de faits plus que dans la narration d'une action dans laquelle s'impliquerait le narrateur.

\section{Conclusion}

Le survol de ces deux récits de voyages castillans permet d'y relever, audelà des dissemblances évidentes, un travail d'écriture commun allant bien plus loin que la description des territoires parcourus ou la chronique froide d'un itinéraire. Le partage des émotions du voyageur au lecteur semble être un outil clef de la stratégie de conviction mise en œuvre dans ces textes qui cherchent à frapper l'imagination. Les contenus didactiques sont ainsi intégrés à la narration du parcours personnel et subjectif des voyageurs. Parfois cette expérience source de savoir mais aussi d'émotions se fige en des tableaux saisissants offerts au lecteur, suspendant le cours du voyage pour mettre en scène un moment clef.

L'hypotypose fonctionne dans ces scènes comme preuve d'authenticité au service d'un effet de réel mais également comme un outil de séduction et un moyen de susciter des émotions. Cette dimension s'affirme en particulier dans les scènes maritimes où la mort menace, véritables morceaux d'anthologie dont s'inspireront les romans d'aventures et de pirates, nous rappelant ainsi l'importance de cet imprévu, de ces forces s'imposant au voyageur, de ce saisissement du lecteur partageant l'angoisse impuissante du héros. L'hypotypose sert à magnifier cette énormité des forces opposées au voyageur, ce surgissement d'un fatum, des circonstances qui le dépassent ${ }^{33}$. L'espace maritime introduisant la menace de la mort, il est aussi celui où le narrateur voyageur est réduit à l'impuissance, laissant l'action aux marins, il ne peut qu'être spectateur de l'action.

Ici se noue l'intérêt de l'hypotypose, cet essentiel « pas de côté » du narrateur devenu spectateur, donnant à voir un tourbillon d'action dans lequel il ne s'engage pas activement. Ce point est commun aux descriptions par Pero Tafur et dans la Embajada des scènes de tempête, spectacles de la force des éléments et des actions des marins, à la cérémonie nautique vénitienne, aux actions des bandits près de Vienne, ou au flot des biens mercantiles à Bruges. Là, on passe de la narration à la mise en scène, au tourbillon accéléré d'une action qui saisit le navire ou le voyageur sans qu'il puisse l'éviter, sans qu'il aie prise sur elle, pour submerger à son tour le lecteur, amené à se mettre en cette position de specta-

\footnotetext{
33 « Un roman d'aventures n'est pas seulement un roman où il y a des aventures ; c'est un récit dont l'objectif premier est de raconter des aventures et qui ne peut exister sans elles. L'aventure est l'irruption du hasard ou du destin dans la vie quotidienne où elle introduit un bouleversement qui rend la mort possible, probable, présente, jusqu'au dénouement qui en triomphe ». J.-Y. Tadié, Le Roman d'aventures, Paris, Quadrige, 1996, p. 5.
} 
teur adoptée par le narrateur. On peut voir dans cette fictionnalisation du récit, dans la vigueur de cette évocation, pour Pero Tafur, le souci de souligner son courage chevaleresque et, pour la Embajada, la volonté de justifier au commanditaire royal l'ampleur des efforts et dangers auxquels furent soumis les ambassadeurs dans leur mission diplomatique. Le récit apparaît comme l'outil d'une quête de reconnaissance matérielle.

Et les valeurs matérielles sont bien présentes dans le récit de Pero Tafur, ce noble atypique aux accointances peu communes. Le lyrisme qu'il déploie pour les descriptions dédiées au commerce, véritable ode à ce titan qui modèle un monde en se riant des distances, est un aveu sans honte de l'intérêt pour les biens et conforts matériels. Cette dimension présente dans bien d'autres passages, et qui n'est pas étrangère au récit de la Embajada, est révélatrice des liens de filiation qui unissent le voyageur et la figure du picaro, ce matérialisme éhonté étant propre à cette figure de anti-héros, jouisseur et amoral.

Les récits de voyages hispaniques ici étudiés révèlent ainsi l'émergence d'une herméneutique moderne dans la constitution d'un savoir empirique, mais aussi dans la rédaction d'une forme narrative dont le héros assume une identité complexe et non idéalisée. Les hypotyposes servent ici à souligner ces aspects du narrateur voyageur parfois impuissant, victime des forces qui le dépassent, ce qui permet également de nouer la sympathie du lecteur à son endroit : tous deux partagent cette position de spectateur d'un monde qui les entraîne et leur impose son rythme et sa fascination.

\section{Bibliographie}

Adam, Jean-Michel et Petitjean, André, Le Texte descriptif : poétique historique et linguistique textuelle, Paris, Nathan-Université, 1989

Boulay, Bérenger, «Effets de présence et effets de vérité dans l'historiographie », Écrire l'histoire, Littérature, $\mathrm{n}^{\mathrm{o}} 159,2010$, p. 26-38 ; URL : http://www.jstor.org/stable/41705312 ; consulté le 26 juillet 2016

Buchet, Christian et Thomasset, Claude (éd.), Le Naufrage. Actes du colloque tenu à l'Institut Catholique de Paris (28-30 janvier 1998), Paris, Honoré Champion, 1999

Calame, Claude, «Quand dire, c'est faire voir, l'évidence dans la rhétorique antique », Études de lettres, Faculté des Lettres, Université de Lausanne, $\mathrm{n}^{\circ} 4$, 1991, p. 3-22

Campbell, Mary B., The Witness and the Other World : Exotic European Travel Writing, 400-1600, Ithaca Cornell University Press, 1988

Cassin, Barbara, L'Effet sophistique, Paris, Nrf, Essais, Gallimard, 1995

Deluz, Christiane, «Pèlerins et voyageurs face à la mer, $\mathrm{XII}^{\mathrm{e}}-\mathrm{XVI}^{\mathrm{e}}$ siècles », in: Horizons marins, itinéraires spirituels, Henri Dubois, Jean-Claude Hocquet et André Vauchez (dir.), t. 2, Marins, navires et affaires, Paris, Sorbonne, 1987, p. 277-288

Fumaroli, Marc, L'Âge de l'éloquence, [Droz, 1980], Paris, Albin Michel, Bibliothèque de l'Évolution de l'Humanité, 1994

Ginzburg, Carlo, Le Fil et les traces. Vrai faux fictif, trad. par Martin Rueff, Lagrasse, Verdier Histoire, 2006 
Herrero Massari, José Manuel, "El naufragio en la literatura de viajes peninsular de los siglos XVI y XVII", Revista de Filología Románica 2.14 (1997), p. 205-213

Larner, John, Marco Polo y el descubrimiento del mundo, Barcelona, Paidós, 2001

Le Bozec, Yves, «L'hypotypose : un essai de définition formelle », L'Information Grammaticale, $n^{\circ}$ 92, 2002, p. 3-7

Le Huenen, Roland «Qu'est-ce qu'un récit de voyages ?, in : Les Modèles du récit de voyages, Litterales $\mathrm{n}^{0} 7,1990$, p. 11-27

López Estrada, Francisco, "Fama literaria de Tamorlán en España durante el siglo XV", in : Studia in Honorem Germán Orduña, Alcalá de Henares, Universidad de Alcalá de Henares, Servicio de Publicaciones, 2001, p. 369-374

Ochoa Anadón, José, "El valor de los viajeros medievales como fuente histórica", Revista de Literatura Medieval 2 (1990), p. 85-102

Pérez Priego, Miguel Ángel (ed.), Andanças e viajes, Fundación José Manuel Lara, clásicos andaluces, Sevilla, 2009

Pérez Priego, Miguel Ángel, (ed.), Viajes medievales, II, Fundación José Antonio de Castro, Madrid, 2006

Richard, Jean, « Voyages réels et voyages imaginaires, instruments de la connaissance géographique au Moyen Âge ", in: idem, Croisés, missionnaires et voyageurs, les perspectives orientales du monde latin médiéval, London, Variorum, 1983, p. 211-220

Richard, Jean, "La relation de voyage, instrument de la transmission du savoir géographique », in : La Transmission des savoirs au Moyen Âge et à la Renaissance, sous la dir. de Pierre Nobel, Presses Universitaires de Franche-Comté, 2005, p. 303-318

Tadié, Jean-Yves, Le Roman d'aventures, Paris, Quadrige, 1996

Traité du sublime, trad. de N. Boileau, éd. de F. Goyet, Le Livre de Poche, 1995

\section{Julia Roumier}

Ancienne élève de l'ENS Lyon, agrégée d'études hispaniques, Julia Roumier est maître de conférences en Civilisation et littérature de l'Espagne classique à l'Université Bordeaux Montaigne. Elle a écrit une thèse de doctorat, qualifiée en histoire médiévale et études hispaniques, et consacrée aux récits de voyages et aux représentations de l'étranger dans le domaine hispanique médiéval. Ses travaux portent sur les récits de voyages et de pèlerinages, la littérature géographique, les chroniques des croisades et sur la cartographie. Auteur de plusieurs articles, elle a publié entre autres « Savoir géographique, encyclopédie et récit de voyage dans le Libro ultramarino (ms 3013 BNE). Traduction et remaniement des textes de Jacques de Vitry et d'Odoric de Pordenone (fin du XIV ${ }^{\mathrm{e}}$ siècle) », in : Le Voyage au Moyen Âge : quête individuelle et description du monde, Christine Gadrat, Damien Coulon (dir.), Presses universitaires de Provence, 2016 ; "La apreciación de lo extranjero: comprensión, elogio y placer en los relatos de viajes medievales cristianos (siglos XIV-XV)", Parnaseo LEMIR Revista de Literatura Española Medieval y del Renacimiento, revue $\mathrm{n}^{\circ} 18,2014$; «La péninsule ibérique et l'Ancien Monde. La littérature de voyages à la fin du $\mathrm{XV}^{\mathrm{e}}$ siècle : connaissance, curiosité, convoitise », Le Verger, Bouquet $\mathrm{n}^{\mathrm{o}}$ 5, 2014. 